\title{
Direct Percutaneous Tracheostomy from NIV in ICU Patients with Respiratory Failure - A Case Series
}

\author{
Rahman L*, Mirja de Vries T, Choo Y Y and Sunwai M \\ Basildon University Hospital, Essex, UK
}

*Corresponding author: Lamay Rahman, Basildon University Hospital, Nethermayne Basildon Essex SS16 5NL, Uk, Tel: 01268 524900; Email: lamay.rahman@btuh.nhs.uk

\section{Case Report \\ Volume 4 Issue 1}

Received Date: March 11, 2019

Published Date: March 19, 2019

\section{Abstract}

Nationally thousands of patients every year require endotracheal intubation after failure of non-invasive ventilation for a multitude of pathologies. These pathologies include but are not limited to chest infections, exacerbations of known lung disease, pancreatitis, neuromuscular diseases and acute respiratory distress syndrome (ARDS). Some of these patients may also be severely haemodynamically unstable and therefore intubation may be associated with a high risk of cardiovascular collapse despite the clinician's best intentions. Patients that will require mechanical ventilation for a prolonged period or be slow to wean off mechanical ventilation can often be accurately predictable. We identified three patients and performed a percutaneous tracheostomy directly from NIV whilst bypassing endotracheal intubation. All 3 of these patients were severely haemodynamically unstable to the extent it was felt that all 3 may completely cardiovascular collapse in the induction or sedative period. However all 3 required invasive ventilation. The procedure was performed under ultrasound guidance, with local anaesthetic infiltration and under light sedation. We describe all three of these patients. The procedure was well tolerated in all 3 cases. Airway care and tracheal suctioning could start immediately after the procedure. As these patients never had to be sedated and ventilated for a prolonged period, no deconditioning occurred. A change to their pre-tracheostomy ventilator settings was not always necessary and weaning could begin promptly. By avoiding the typical 7-10 day period of intubation and deconditioning, it is approximated ITU stay could be cut by up to 7 days. This can have a significant economic impact in both ITU bed days as well as physical capacity. We devised a safety checklist to maintain safety during the procedure, advocating the use of ultrasound whilst remaining aware at all times of the potential urgent need for airway protection.

Keywords: Tracheostomy; Haemodynamically; Anaesthetic; Lidocaine; Ventilatory 


\section{Anaesthesia \& Critical Care Medicine Journal}

Abbreviations: THRIVE: Trans Nasal Humidified Rapid-Insufflation Ventilatory Exchange; NIV: NonInvasive Ventilation; ITU: Intensive Treatment Unit.

\section{Background}

Severe acute respiratory infections account for one in ten of all intensive care admissions. These patients have a high rate of organ failure (74.7\%), particularly respiratory failure $(53.8 \%)$, as well as high in-hospital mortality rates $(27.2 \%)$ [1].

These patients are initially treated with non-invasive techniques, including supplementary oxygen, Trans nasal Humidified Rapid-Insufflation Ventilatory Exchange (THRIVE) and/or non-invasive ventilation (NIV). Patients who develop worsening respiratory failure often go on to require invasive ventilation as the non-invasive techniques do not meet the necessary respiratory requirements. A recent study suggests this NIV failure rate may be as high as in $30 \%$ of cases. After intubation, a high number of patients, often quite predictably, require long term ventilation and a tracheostomy becomes indicated [2-5].

Those that fail NIV and require intubation can at times be haemodynamically unstable due to current pathology married to pre-existing co-morbidities. These patients are at risk of cardiovascular collapse at time of induction for intubation or soon after intubation with the intubation associated increased sedative requirements.

A Cochrane meta-analysis looking at the benefits of having an early tracheostomy suggested a possible superiority of early intervention, with reduced mortality and increased rate of discharge from the intensive care unit at day 28 [6].

\section{Methods}

\section{Patient Selection}

A selection of 3 patients was made upon which there could be a reliable predication that, if intubated, the patients would require mechanical ventilation for a number of weeks and/or be slow to be able to be weaned off mechanical ventilation and Therefore would require a tracheostomy in the near future post intubation. All three of these patients were also significantly hemodynamically unstable to the degree it was felt that he may go into complete cardiovascular collapse if they were to be intubated.

\section{Procedure}

In all 3 cases, the patients themselves were able to consent for the percutaneous tracheostomy to be carried out under local anaesthetic with light sedation only. In 2 of the cases the family was also informed and consented about the procedure.

After adequate preparation as described by the checklist in table 1, the pre-tracheal anatomy was visualized using ultrasound, with special note taken of blood vessels that may block any potential path of the tracheostomy from the skin to the trachea. The patients were pre-oxygenated with $100 \%$ oxygen via the NIV device they were already being ventilated with. 2 of the patients were given $2 \mathrm{mg}$ Midazolam prior to starting the procedure and 1 patient did not require any sedation.

Under aseptic conditions, after identifying the site of insertion, local infiltration was made with $10 \mathrm{mls}$ of lidocaine $1 \%$ with adrenaline 1:200,000. The tracheostomies were inserted via the conventional single tapered dilatational seldinger technique and ventilation confirmed by the tracing of end tidal carbon dioxide.

We are unaware of any tracheostomies being done in this setting elsewhere, so three cases are described here.

\section{Patient 1}

A 63-year old gentleman with no co-morbidities was admitted to hospital with progressive four-limb weakness, blurred vision in his right eye and diplopia following a flulike illness two weeks previous. He was areflexic and had a type 1 respiratory failure. An MRI brain was carried out acutely and showed small vessel ischemia with a subacute lacunar infarct. A diagnosis of probable Miller Fisher syndrome was made and he was commenced on intravenous piperacillin/tazobactam and immunoglobulin's. Whilst on treatment on the medical ward, his breathing detiorated rapidly and so was admitted to ITU for NIV. He became septic soon after admission to ITU and required high amounts of inotropic support to maintain a mean arterial blood pressure. During this period he remained fully awake and orientated. On day 3 of his ITU stay, he became increasingly cardiovascular unstable and required escalating Ventilatory support. Due to the concern as to whether he would tolerate being induced and ventilated, it was decided, with his consent, to do a percutaneous tracheostomy whilst still on NIV, under lidocaine/adrenaline infiltration and no sedation. The 


\section{Anaesthesia \& Critical Care Medicine Journal}

tracheostomy was carried out with nil complications and he remained on the same NIV settings post tracheostomy.

He developed complications during his ITU stay, including completely blindness and total flaccidity. However, by day 30 , he started to improve, his vision returned and he was tolerating small periods of time on CPAP. He continued to show stepwise improvement. His tracheostomy was capped on day 66 and he was discharged to the ward on day 69. A week later, his tracheostomy was removed completely. He was eventually discharged to the community hospital, with successful discharge home 144 days after his initial presentation

\section{Patient 2}

A 86-year old gentleman with a background of asthma, chronic obstructive pulmonary disease, chronic kidney disease, a previous stroke with residual left sided weakness, hypertension, and a small abdominal aortic aneurysm presented to the emergency department from a community hospital with shortness of breath and coughing just four days after discharge from hospital where he had been treated for pneumonia and an acute kidney injury. He was a smoker and had a poor exercise tolerance.

In A\&E he was found to have a type 1 respiratory failure, a new acute kidney injury and was haemodynamically unstable. He was transferred to ITU where he was treated for pneumonia. He was started on NIV and commenced on inotropic support. He remained conversant but hypoxic despite a high FiO2 with NIV. His respiratory failure worsened and he required invasive ventilation. The family was very keen to continue treatment. It was felt that there was a significant chance that he may completely cardiovascular collapse at intubation due to the high amount of inotropic support he was on. With his and the family's consent, a decision was made to perform a percutaneous tracheostomy whilst on NIV under local anaesthetic. He was also given two milligrams midazolam. The procedure was uneventful, and the patient remained haemodynamically stable and without any increase in ventilator settings or inotropic requirements. The next day his chest began to improve, and by day 6 of his admission, he was weaned off noradrenaline and his oxygen requirements were coming down.

He had a prolonged stay in intensive care due to multiple chest infections, and a non-ST segment elevated myocardial infarction. By day 20, he was breathing on a Tpiece, and gradually weaned off the ventilator. He was discharged to the ward 32 days after his ICU admission.

\section{Patient 3}

A 56-year old gentleman with a background of acute myeloid leukemia, bone marrow transplants, and venous thrombosis, was admitted to hospital with severe headaches and scalp tenderness. Investigations including a CT head, MRI head and CT chest showed no abnormalities. The patient developed hospital acquired pneumonia with a type 1 respiratory failure, for which he received THRIVE therapy.

He was admitted to ICU for NIV due to increasing oxygen requirements after 13 days on the ward. He was diagnosed with Ramsey Hunt syndrome and varicella zoster viral pneumonitis, and was commenced on treatment with acyclovir, meropenem, clarithromycin, penicillin $\mathrm{V}$ and prednisolone. He showed no improvement on intermitted NIV and THRIVE. Despite his $\mathrm{FiO}_{2}$ requirements increasing, he remained alert and orientated, but was very NIV dependent. As he was able to cope with his breathing, he refused to be ventilated. There was no improvement for the next 5 days. On day 6, his breathing pattern looked significantly worse than the previous 5 days and this time he accepted being put on a ventilator. He was on very high doses of inotropic support and high NIV settings with a $\mathrm{FiO}_{2}$ of $80 \%$. With his wife's and his consent, a percutaneous tracheostomy was performed on day 8 under local lidocaine/adrenaline infiltration and sedation using two milligrams of midazolam. The tracheostomy was inserted with no complications and he was given the higher ventilator support he required.

He had relapsing episodes of hypoxia with increased secretions during the following hours. However, by day 11 he was able to breathe through a T-piece. After some other complications, he began to improve and was fit for ward discharge on day 23. His tracheostomy tube was capped on day 25. He finally left hospital after 92 days, due to some complications caused by thrombocytopenia, pseudomonas infection and right vocal cord palsy.

\section{Discussion}

Conventionally, tracheostomies have been inserted to facilitate weaning from mechanical ventilation. In some circumstances, patients that have been very slow or difficult to wean off mechanical ventilation with an 


\section{Anaesthesia \& Critical Care Medicine Journal}

endotracheal tube are able to be weaned promptly after having a tracheostomy in place.

Having a tracheostomy in place as compared to an endotracheal tube means that less sedation is required to tolerate the airway, a big advantage in patients that may already be severely haemodynamically unstable. Tracheostomies have the advantage of allowing easier and better suctioning and airway care, which may also reduce the rate of ventilator associated pneumonias [7]. They also provide less dead space compared to an endotracheal tube and reduce the resistance to breathing. Physiotherapy is normally much better tolerated and easier with a tracheostomy [8].

Intubation in itself has been linked to a number of complications due to damage to the oropharynx and larynx. These complications occur more frequently and are more pronounced the longer the patient has been intubated. With a patient that is sedated and intubated for 7-10 days, there may be periods in that timescale where the sedation the patient is on is not enough to prevent movements of the neck, leading to potential for both frictional and mechanical damage to the oropharynx and larynx [9].

Comparing a tracheostomy to non-invasive ventilation means that enteral feeding can be started immediately as there is a definitive secure airway in place and so the benefits of having a fed patient can be utilized. Higher and more effective Ventilatory support can be provided if necessary via a tracheostomy compared to non-invasive methods.

As highlighted in the above 3 cases, we bypassed the intubation stage in these patient's conventional treatment strategies and performed a tracheostomy whilst the patient was still on non-invasive ventilation. We found that this procedure to be well tolerated in all 3 cases, with only $2 \mathrm{mg}$ Midazolam required for 2 patients and no sedation at all required for 1 patient. 10mls Lidocaine 1\% with 1:200,000 adrenalines was sufficient in all 3 cases as the quantity and strength of local anaesthetic. .

In two of the cases the patients were able to remain on the same Ventilatory settings prior to and post tracheostomy as no de-conditioning occurred compared to those who have been ventilated for a few days prior to their tracheostomy. Airway care and feeding started immediately, and weaning commenced very soon afterwards. In the third case, the tracheostomy allowed him to receive the higher Ventilatory support he needed compared to NIV, as well as regular suctioning, airway care, feeding and physiotherapy.

All 3 patients described above had severe respiratory failure and were on high amounts of inotropic support. All 3 needed invasive Ventilatory support. It was felt that all 3 may not have survived the peri-intubation period due to need for sedation and increased PEEP coupled with an already increasing inotropic requirement. Had they survived, they would have taken a longer time for weaning to start due to muscle de-conditioning whilst intubated.

The advantage of this technique is that by bypassing the period of endotracheal intubation prior to tracheostomy, the patients did not de-condition whilst remaining stable on the ventilator. Additionally this allows them to receive earlier support, including regular suctioning, feeding and physiotherapy from the moment the tracheostomy was inserted, thus speeding up their recovery and most likely saving an approximation of one week in intensive care whilst intubated. This may have major cost saving implication on ITU bed days.

We found the procedure to be well tolerated. It gives a new option for patients where uncertainty exists regarding hemodynamic stability during intubation and sedation, and a potential increase in Ventilatory support immediately post intubation. We remained aware at all times of the potential need for intubation and were prepared for that.

To our knowledge, this procedure has not been described before. Therefore, we have devised a safety checklist for the procedure (Table 1), allowing the procedure to be performed safely in the hands of an experienced operator, without the need for endotracheal intubation or endoscopic assistance. We found that use of ultrasound a necessity and an improvement as a substitute for the fiberscope [10]. 


\section{Anaesthesia \& Critical Care Medicine Journal}

Checklist for percutaneous tracheostomy without intubation

Patient consented and/or procedure discussed with family

Contra-indications ruled out

Skilled assistant present

Equipment for procedure ready:

- Aseptic tracheostomy set including scalpel, guidewire, dilators, tracheostomy tube

- $\quad$ bag mask

- $\quad$ oxygen supply

- ventilator

- ultrasound scanning machine

- $\quad$ benzodiazepine for intravenous sedation if using

- $\quad$ lidocaine $1 \%$ with adrenaline 1:200 000

- $\quad$ end tidal carbon dioxide monitoring

Intubation equipment available and ready:

- laryngoscopes

- $\quad$ endotracheal tubes in appropriate sizes

- bougie

- $\quad$ anaesthetic agent, suxamethonium and muscle relaxant available

- $\quad$ emergency drugs available (vasopressors/inotropes/anticholinergic/reversal agents)

Laryngeal mask airway available

Patient positioned correctly

Appropriate monitoring in place

Pre-tracheal anatomy, especially blood vessels, identified/confirmed using ultrasound

Pre-oxygenation with $100 \%$ oxygen

Existing ventilatory support continued (i.e. non-invasive ventilation)

Sedation given if appropriate

Procedure performed by an experienced operator

End Tidal carbon dioxide to confirm ventilation

Chest X-ray to confirm position

Table 1: Checklist for percutaneous tracheostomy without intubation.

\section{References}

1. Sakr Y, Ferrer R, Reinhart K, Beale R, Rhodes A, et al. (2016) The Intensive Care Global Study on Severe Acute Respiratory Infection (IC-GLOSSARI): a multicenter, multinational, 14-day inception cohort study. Intensive Care Med 42(5): 817-828.

Rahman L, et al. Direct Percutaneous Tracheostomy from NIV in ICU Patients with Respiratory Failure - A Case Series. Anaesth Critic Care Med J 2019, 4(1): 000146.
2. Ranes JL, Gordon SM, Chen P, Fatica C, Hammel J, et al. (2006) Predictors of long-term mortality in patients with ventilator-associated pneumonia. The American Journal of Medicine 119(10): 819.e13-e19.

3. Holzapfel L, Chevret S, Madinier G, Ohen F, Demingeon G, et al. (1993) Influence of long-term 


\section{Anaesthesia \& Critical Care Medicine Journal}

oro- or nasotracheal intubation on nosocomial maxillary sinusitis and pneumonia: results of a prospective, randomized, clinical trial. CritIcal Care Medicine 21(8): 1132-1138.

4. Cavaliere S, Bezzi M, Toninelli C, Foccoli P (2007) Management of post-intubation tracheal stenoses using the endoscopic approach. Monaldi Archives for Chest Disease 67(2): 73-80

5. Corrêa TD, Sanches PR, de Morais LC, Scarin FC, Silva E, et al. (2015) Performance of noninvasive ventilation in acute respiratory failure in critically ill patients: a prospective, observational, cohort study. BMC Pulm Med 15: 144.

6. Andriolo BNG, Andriolo RB, Saconato H, Atallah ÁN, Valente $O$ (2015) Early versus late tracheostomy for critically ill patients. Cochrane Database of Systematic Reviews 1: CD007271.
7. Nseir S, Pompeo C, Jozefowicz E, Cavestri B, Brisson $\mathrm{H}$, et al. (2007) Relationship between tracheotomy and ventilator-associated pneumonia: a case control study. Durocher European Respiratory Journal 30: 314-320.

8. Durbin CG (2005) Indications for and timing of tracheostomy. Respir Care 50(4): 483-487.

9. Mota LA, de Cavalho GB, Brito VA (2012) Laryngeal complications by orotracheal intubation: Literature review. Int Arch Otorhinolaryngol 16(2): 236-245.

10. Gobatto A, Besan B, Luiz Malbouisson (2016) Ultrasoung-guided percutaneous dilational trachaeostomy $\mathrm{v} / \mathrm{s}$ bronchoscopy guided percutaneous dilational trachaeostomy in critically ill patients (TRACHUS): a randomized noninferiority controlled trial. Intensive Care Med 42(3): 342-351. 